\title{
Boosting Escherichia coli's heterologous production rate of ectoines by exploiting the non-halophilic gene cluster from Acidiphilium cryptum
}

\author{
Lukas Bethlehem $^{1} \cdot$ Katharina D. Moritz ${ }^{1}$
}

Received: 28 April 2020 / Accepted: 6 July 2020 / Published online: 22 July 2020

(c) The Author(s) 2020

\begin{abstract}
The compatible solutes ectoine and hydroxyectoine are synthesized by many microorganisms as potent osmostress and desiccation protectants. Besides their successful implementation into various skincare products, they are of increasing biotechnological interest due to new applications in the healthcare sector. To meet this growing demand, efficient heterologous overproduction solutions for ectoines need to be found. This study is the first report on the utilization of the non-halophilic biosynthesis enzymes from Acidiphilium cryptum DSM $2389^{\mathrm{T}}$ for efficient heterologous production of ectoines in Escherichia coli. When grown at low salt conditions $(\leq 0.5 \% \mathrm{NaCl})$ and utilizing the cheap carbon source glycerol, the production was characterized by the highest specific production of ectoine $[2.9 \mathrm{~g} / \mathrm{g}$ dry cell weight $(\mathrm{dcw})]$ and hydroxyectoine $(2.2 \mathrm{~g} / \mathrm{g} \mathrm{dcw})$ reported so far and occurred at rapid specific production rates of up to $345 \mathrm{mg} /(\mathrm{g} \mathrm{dcw} \times \mathrm{h})$. This efficiency in production was related to an unprecedented carbon source conversion rate of approx. $60 \%$ of the theoretical maximum. These findings confirm the unique potential of the here implemented non-halophilic enzymes for ectoine production processes in $E$. coli and demonstrate the first efficient heterologous solution for hydroxyectoine production, as well as an extraordinary efficient low-salt ectoine production.
\end{abstract}

Keywords Compatible solutes $\cdot$ Hydroxyectoine $\cdot$ Ectoine $\cdot$ Industrial applications $\cdot$ Escherichia coli $\cdot$ Acidiphilium cryptum

\section{Introduction}

Compatible solutes are small organic compounds, which are accumulated or synthesized by microorganisms under physical stress conditions enabling protection of whole cells and macromolecules against, for example, high osmolarity, heat, freezing, and desiccation (Manzanera et al. 2004; Borges et al. 2002; Göller and Galinski 1999; Kempf and Bremer

Communicated by M. Moracci.

Lukas Bethlehem and Katharina D. Moritz contributed equally.

Electronic supplementary material The online version of this article (https://doi.org/10.1007/s00792-020-01188-8) contains supplementary material, which is available to authorized users.

Lukas Bethlehem

ifmb@uni-bonn.de

1 Institute for Microbiology and Biotechnology, University Bonn, Meckenheimer Allee 168, 53115 Bonn, Germany
1998; Louis et al. 1994; Lippert and Galinski 1992). For this purpose, they can be enriched up to molar concentrations in a cell without adverse effects on its metabolism (Brown 1976). Ectoine and hydroxyectoine are derivatives of the amino acid aspartate and are widely abundant osmo- and heat-protectants in halophilic microorganisms. Both compounds share potent moisturizing and skin protective features, which led to implementations of especially ectoine into a multitude of skincare and well-being products (Pastor et al. 2010). Compared to ectoine, hydroxyectoine displays even higher water-binding activities and enhanced glassforming attributes, making this compatible solute a potent desiccation protectant (Manzanera et al. 2004; Tanne et al. 2014). Furthermore, ectoine and hydroxyectoine are distinct in their effect on DNA. While ectoine lowers the melting temperature of double-stranded DNA, hydroxyectoine conversely elevates it (Seip et al. 2011). Recently, the field of application is expanding due to studies demonstrating antiinflammatory effects of ectoine and ectoine derivatives in intestinal bowel disease and carbon nanoparticle induced 
airway inflammation, as well as protein stabilizing effects in neurodegenerative diseases (Abdel-Aziz et al. 2013, 2015; Bazazzadegan et al. 2017; Unfried et al. 2014). Promising applications as adjuvant treatment in these diseases led to a gain in CAGR (calculated annual growth rate) for the ectoine market and therefore to a demand for feasible biotechnological production processes (Becker and Wittmann 2020).

Ectoine is currently produced and marketed at large scale primarily utilizing the natural producer strain Halomonas elongata and optimized strain derivatives of this organism (Kunte et al. 2014). In contrast to ectoine, hydroxyectoine production in $H$. elongata is only possible under substantial accumulation of its precursor ectoine (Wohlfarth et al. 1990). As a consequence, purification of hydroxyectoine would require a time- and cost-intensive chromatographic separation of both solutes, thus restricting relevant current applications to ectoine only. A viable hydroxyectoine production process would allow to screen for new applications with this compound due to the distinctive features of hydroxyectoine. However, also the production of ectoine in H. elongata is a cost-intensive process, as it demands high concentrations of sodium chloride $(\mathrm{NaCl})$, which makes expensive desalting and salt disposal during downstream processing a necessary requirement.

To meet the demand for more efficient production of ectoines (= ectoine + hydroxyectoine), increasing numbers of heterologous production strains have been generated in recent years that enable an extracellular accumulation of ectoines at low $\mathrm{NaCl}$ concentrations (Parwata et al. 2019; Gießelmann et al. 2019; Czech et al. 2018; Pérez-García et al. 2017; Ning et al. 2016; He et al. 2015; Eilert et al. 2013; Becker et al. 2013; Schubert et al. 2007). Interestingly, the employed biosynthesis enzymes for heterologous production of ectoines originated from halophilic donors with optimum activities in the presence of salts. This might be a reasonable approach for ectoine production in the Gram-positive host Corynebacterium glutamicum, which naturally exhibits a relatively high ionic strength in its cytoplasm (Krämer et al. 1990; Follmann et al. 2009). In the Gram-negative host Escherichia coli, however, activities of halophilic enzymes could be significantly impaired under low salt conditions, due to the lower ionic strength in its cytoplasm (Dinnbier et al. 1988). In this study, we therefore used the biosynthesis gene cluster from the non-halophilic

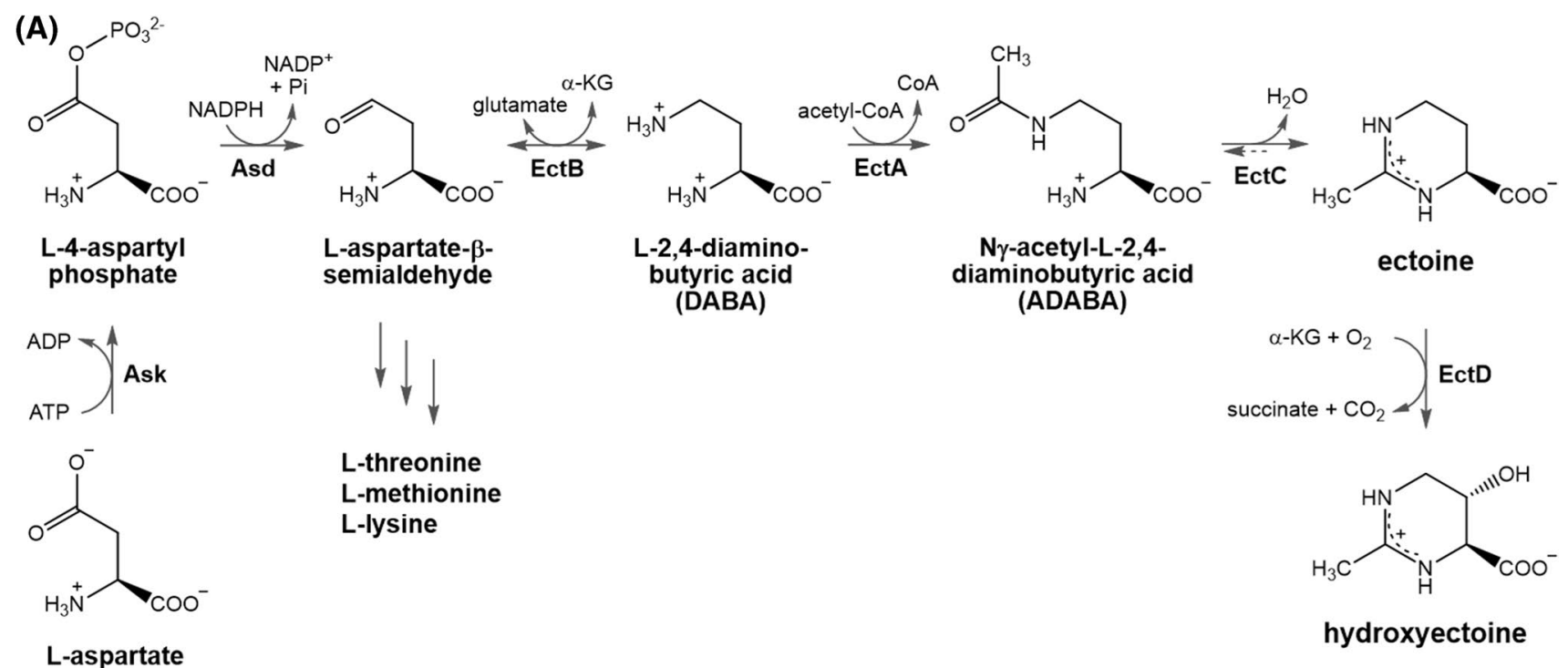

(B)

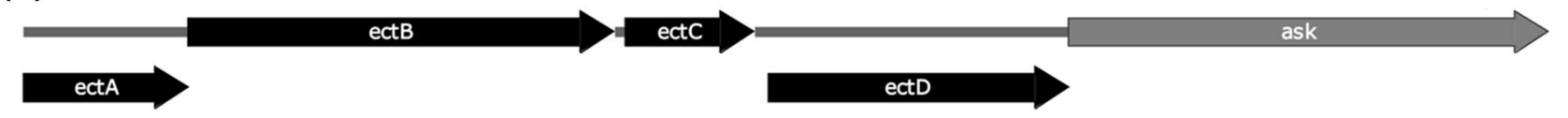

Fig. 1 Pathway of ectoine and hydroxyectoine biosynthesis. a Biosynthesis of ectoine and hydroxyectoine starts with the amino acid L-aspartate. L-aspartate- $\beta$-semialdehyde describes a joint precursor for the aspartate amino acid family and ectoines. Ask aspartokinase, Asd aspartate- $\beta$-semialdehyde dehydrogenase, EctB L-2,4-diaminobutyric acid transaminase, EctA L-2,4-diaminobutyric acid acetyl- transferase, $E c t C$ ectoine synthase, $E c t D$ ectoine hydroxylase, $\alpha-K G$ $\alpha$-ketoglutarate, CoA Coenzyme A. b The A. cryptum hydroxyectoine biosynthesis gene cluster comprises the genes for acetyltransferase (ectA), transaminase (ectB), ectoine synthase (ect $C)$, ectoine hydroxylase (ectD), as well as an additional aspartokinase gene (ask) overlapping ectD 
organism Acidiphilium cryptum for the heterologous production of ectoines in E. coli.

The biosynthetic pathway of ectoine and hydroxyectoine is well-studied (Peters et al. 1990; Bursy et al. 2007; Czech et al. 2019) and branches from the biosynthesis of the aspartate amino acid family (Fig. 1a). The transaminase EctB catalyses the transamination of the precursor L-aspartate$\beta$-semialdehyde to L-2,4-diaminobutyric acid (DABA). The following acetylation to $\mathrm{N} \gamma$-acetyl-L-2,4-diaminobutyric acid (ADABA) is carried out by the acetyltransferase EctA, followed by the cyclization to ectoine catalysed by the ectoine synthase EctC. For the conversion to hydroxyectoine, the ectoine ring is hydroxylated by the ectoine hydroxylase EctD. Usually, these biosynthetic genes are organized in one operon, which is in some organisms extended by an ask gene coding for an additional aspartokinase. This enzyme catalyses the second step in the pathway of the aspartate amino acid family, phosphorylating L-aspartate to L-4-aspartyl phosphate (Fig. 1a). This step can be a critical bottleneck for the ectoine biosynthesis, due to a possible feedback inhibition of the Ask protein by the end products of the aspartate amino acid family (threonine, methionine, lysine) (Bestvater et al. 2008). Such an additional ask gene is also present in the here used hydroxyectoine biosynthesis gene cluster of $A$. cryptum DSM $2389^{\mathrm{T}}$ overlapping the ectD gene (Fig. 1b).

A. cryptum is an acidophilic $\alpha$-proteobacterium which thrives in acidic, metal-rich environments (Harrison 1981). It was shown that $A$. cryptum is capable of producing the compatible solute hydroxyectoine in response to elevated $\mathrm{NaCl}$ or $\mathrm{Al}_{2}\left(\mathrm{SO}_{4}\right)_{3}$ levels, despite the organism's rather weak salt tolerance of up to $5 \% \mathrm{NaCl}$ (Moritz et al. 2015). The A. cryptum hydroxyectoine biosynthesis proteins differ from halophilic variants by their less acidic nature implying optimum activity in the absence of salt, as experimentally shown for the EctC enzyme activity (Moritz et al. 2015). We therefore hypothesized, that this capacity could enable more efficient overproduction of ectoines at low salt conditions in non-halophilic host organisms with a low ionic strength in their cytoplasm. In this study, the productivity of the unique biosynthesis proteins of A. cryptum was tested for the first time under optimized conditions in $E$. coli in order to prove their potential for the heterologous hydroxyectoine and ectoine production under low salt conditions.

\section{Materials and methods}

\section{Bacterial strains, media, and cultivation}

All strains in this study were acquired from the DSMZ (Braunschweig, Germany). The type strain A. cryptum DSM $2389^{\mathrm{T}}$ (Harrison 1981) was used as the donor for the hydroxyectoine biosynthesis gene cluster. Cultivation was performed in modified M269 medium (Harrison 1981), containing (in g/L): $\left(\mathrm{NH}_{4}\right)_{2} \mathrm{SO}_{4}(2.0), \mathrm{KCl}(0.1), \mathrm{K}_{2} \mathrm{HPO}_{4}$ (0.5), $\mathrm{MgSO}_{4} \times 7 \mathrm{H}_{2} \mathrm{O}(0.5)$, yeast extract (0.3), glycerol (5.0). The strain E. coli DH5 $\alpha$ DSM 6897 (Hanahan 1983) $\left(\mathrm{F}^{-} \varphi 80\right.$ lacZDM15 $\Delta($ lacZYA-arg $F) \mathrm{U} 169$ deoR recA1 endA1 hsdR $17\left(\mathrm{rk}^{-} \mathrm{mk}^{+}\right)$phoA supE $44 \lambda^{-}$thi-1 gyrA96 relA1) was used as the host for transformation experiments as well as for hydroxyectoine and ectoine overproduction experiments. For cloning steps, E. coli DH5 $\alpha$ was cultivated in LB medium (Bertani 1951). For overproduction of ectoines the defined medium MM63 was utilized (Larsen et al. 1987), containing (in g/L): $\mathrm{KH}_{2} \mathrm{PO}_{4}(13.61$ ), $\mathrm{KOH}(4.21),\left(\mathrm{NH}_{4}\right)_{2} \mathrm{SO}_{4}(1.98), \mathrm{MgSO}_{4} \times 7 \mathrm{H}_{2} \mathrm{O}(0.25)$, $\mathrm{FeSO}_{4} \times 7 \mathrm{H}_{2} \mathrm{O}$ (0.0011), and glucose-monohydrate (5.0) or glycerol (4.65). $\mathrm{NaCl}$ was added to the medium in concentrations of $0-1 \%$ and is stated if relevant. A vitamin solution was added to enable growth of E. coli DH5 $\alpha$ (Imhoff and Trüper 1977), resulting in final concentrations (in $\mathrm{g} / \mathrm{L}$ ): nicotinamide (0.35), Ca-DL-pantothenate $(0.10)$, thiamine $\times \mathrm{HCl}(0.30), p$-aminobenzoic acid (0.20), pyridoxal chloride (0.10), cyanocobalamin (0.05), biotin (0.10). For plasmid maintenance, $100 \mathrm{mg} / \mathrm{L}$ of the antibiotic carbenicillin were added. All cultivations were performed aerobically at $37^{\circ} \mathrm{C}$ and $180 \mathrm{rpm}$ in $250 \mathrm{~mL}$ shake flasks with baffles.

\section{Construction of plasmids}

For the heterologous production of ectoines in E. coli utilizing the hydroxyectoine biosynthesis gene cluster from $A$. cryptum, plasmids based on the pASK-IBA3 vector (IBA Lifesciences, Göttingen, Germany) were constructed. The vector comprises a bla gene, a ColE1 replicon, and a tet promoter, which enables high expression levels after induction with anhydrotetracycline (AHT). Plasmid constructions were performed by standard restriction cloning, and plasmids were integrated into chemically competent $E$. coli $\mathrm{DH} 5 \alpha$ cells via transformation. The sequencing of plasmids was carried out by Eurofins Genomics (Ebersberg, Germany). Two different plasmids were designed, containing either ect$A B C D a s k$ or ectABCD, allowing to compare hydroxyectoine production with and without the additional aspartokinase from A. cryptum (Table 1; Figure S1, Supplementary material). The plasmid pASK_ectABCDask was constructed in a two-step cloning process using the PstI restriction site present in $e c t D$ to ligate both fragments. Construction of pASK_ectABCD was performed in one-step cloning. The plasmid pASK_ectABCD ${ }_{m}$ was found to result in ectoine production due to a random point mutation in $e c t D$ and was used for most ectoine overproduction processes in this study. The plasmid pASK_ectABC was constructed in a one-step restriction cloning after detection of the random mutagenesis 
Table 1 Information on plasmid constructions

\begin{tabular}{|c|c|c|c|c|}
\hline Construct & & Label & Nucleotide sequence $\left(5^{\prime}-3^{\prime}\right)$ & Restriction site \\
\hline \multirow[t]{4}{*}{ ectABCDask } & Step 1 & fwd1 & ATTTCTAGACGGTGGCGCTGCGTC & XbaI \\
\hline & & rev1 & CCGACTGCAGCAGCGCGATCT & PstI \\
\hline & Step 2 & fwd2 & AGATCGCGCTGCTGCAGTCG & PstI \\
\hline & & rev2 & ATTAAGCTTCGCCCGCATGCACGA & HindIII \\
\hline \multirow{3}{*}{$\begin{array}{l}\text { ectABCD, } \\
\text { ectABCD } \\
\text { ectABC }\end{array}$} & & fwd1 & & \\
\hline & & rev3 & ATTAAGCTTTCGGAGCCATGCTCATGC & HindIII \\
\hline & & $\begin{array}{l}\text { fwdl } \\
\text { rev4 }\end{array}$ & AATAAGCTTCGTCAGGCCGCCTCGCCGA & HindIII \\
\hline \multirow[t]{8}{*}{ ectAB-RectC } & Step 1 & fwd3 & GTGCATATGATCATCCGGACTCTGAAGGAG & NdeI \\
\hline & & rev5 & ATTCTCGAGGGCCGCCTCGCCGACC & XhoI \\
\hline & Step 2 & fwd4 & TAATACGACTCACTATAGG & \\
\hline & & rev6 & ATTAAGCTTGCGGTGGCAGCAGCCAACT & HindIII \\
\hline & Step 3 & fwd5 & ATTCCATGGTGGCCAGATGATTAATTCCT & NcoI \\
\hline & & rev7 & ATTAAGCTTTCAGGCCGCCTCGCCGA & HindIII \\
\hline & Step 4 & fwd1 & & \\
\hline & & rev8 & ATTCCATGGCAGAGTCCGGATGATCATG & NcoI \\
\hline
\end{tabular}

Primers used for restriction cloning of hydroxyectoine (ectABCDask, ectABCD) and ectoine (ect $\mathrm{ABCD}_{\mathrm{m}}$, ect $\mathrm{ABC}$, ect $\mathrm{AB}-\mathrm{RectC}$ ) overproduction plasmids are listed. Restriction sites are underlined to exclude any detrimental effects of $e c t D_{\mathrm{m}}$ on ectoine production (Table 1). The plasmid pASK_ectAB-RectC was designed in a multiple cloning process to assess the effect of ectC overexpression on ADABA accumulation during ectoine overproduction (Table 1; Figure S2, Supplementary material). In this case, the expression of ectC is under the control of a separate tet promoter and the optimized ribosome binding site (RBS) from the pET-22b(+) vector.

\section{Overproduction, detection, and quantification of ectoines}

Precultures of the production strains were prepared in the same medium as the main cultures and incubated overnight at $37^{\circ} \mathrm{C}$ and $180 \mathrm{rpm}$. The preculture was centrifuged at $4500 \times g$ and cells were resuspended in fresh medium. Main cultures (100 mL MM63) were inoculated to an optical density at $600 \mathrm{~nm}\left(\mathrm{OD}_{600}\right)$ of 0.1 and induced at $\mathrm{OD}_{600}$ of $0.3-0.4$ by adding $0.2 \mathrm{mg} / \mathrm{L}$ AHT. Cells were harvested in the stationary phase and freeze-dried by lyophilization for calculation of the dry cell weight (dcw). To determine the intracellular solute concentration, the freeze-dried cells were extracted using a modified Bligh and Dyer protocol (1959), as described previously (Galinski and Oren 1991). The water-soluble fraction and the culture supernatant were analysed by isocratic high-performance liquid chromatography (HPLC) using a LiChrospher amino phase column (Merck, Darmstadt, Germany) and acetonitrile/water (80/20\% v/v) as the mobile phase. Measurements were carried out at ambient temperature and a flow rate of $1 \mathrm{~mL} / \mathrm{min}$. For quantification of ectoine and hydroxyectoine, UV and refractive index detection were employed as described by Galinski and Herzog (1990). The overproduction experiments were carried out in at least triplicates $(n=3-20)$ and the results displayed with standard deviation. The specific production rate over time is given as mean with upper/lower boundary $(n=2)$.

\section{Detection and quantification of by-products and carbon sources}

For detection of zwitterionic and uncharged by-products (e.g. trehalose), the culture supernatant was analysed by isocratic HPLC as described for detection of ectoines. Quantification of glycerol, glucose, and acetate in the culture medium was performed by isocratic HPLC with an Aminex HPX-87H column (Bio-Rad, Hercules, USA). As a mobile phase, $0.02 \mathrm{~N} \mathrm{H}_{2} \mathrm{SO}_{4}$ was used at a flow rate of $0.6 \mathrm{~mL} /$ min. For eluent detection, UV and refractive index detection were employed. Furthermore, N-reactive compounds in the culture supernatant were analysed by gradient HPLC with pre-column FMOC-ADAM-derivatization as described previously (Kunte et al. 1993). The concentration of the precursor ADABA was determined by this method. 


\section{Carbon balance calculation}

The carbon balance calculation was carried out for the production strains based on overproduction experiments under optimum growth conditions, with the carbon source entirely consumed and no other products than ectoines detected in the culture medium. The control strain E. coli DH5 $\alpha$ with the empty vector (pASK-IBA3), which generates only biomass under the same conditions, was used to elucidate the carbon input for the heterologous production of ectoines separately from the biomass formation. In the case of hydroxyectoine production, the ectoine share of $3.6 \%$ was not considered when calculating the carbon balance.

\section{Protein expression and separation via SDS-PAGE}

A protein expression experiment was performed to visualize the production of the heterologous proteins in the constructed E. coli strains. The cells were cultivated as described before and protein expression was induced by the addition of AHT $(0.4 \mathrm{mg} / \mathrm{L})$. Before and after induction, $10 \mathrm{~mL}$ samples were taken for protein analysis. The cell pellets were processed in a standard protein extraction with lysozyme and sodium dodecyl sulfate (SDS). After separating the soluble protein fraction by centrifugation at $4{ }^{\circ} \mathrm{C}$ and $16,000 \times g$ for $30 \mathrm{~min}$, its protein content was determined by the bicinchoninic acid assay after Smith et al. (1985). The soluble protein extracts were analysed semi-quantitative by SDS polyacrylamide gel electrophoresis (SDS-PAGE) after Laemmli (1970). In the case of E. coli DH5 $\alpha$ pASK_ect$\mathrm{ABCD}_{\mathrm{m}}$ and pASK-IBA3 $20 \mu \mathrm{g}$ of soluble protein extracts were loaded on the gel, and in the case of E. coli DH5 $\alpha$ pASK_ectAB-RectC $10 \mu \mathrm{g}$ of soluble proteins were applied.

\section{Results}

\section{Hydroxyectoine production}

For hydroxyectoine production the strain E. coli $\mathrm{DH} 5 \alpha$ pASK_ectABCDask was used, expressing the entire hydroxyectoine biosynthesis gene cluster from $A$. cryptum under control of the AHT-inducible tet promoter. For overproduction experiments, the strain was cultivated in shake flasks utilizing minimal medium with a low $\mathrm{NaCl}$ concentration $(0-1 \%)$ and either $25 \mathrm{mM}$ glucose or $50 \mathrm{mM}$ glycerol as carbon source. The growth rate of cultures increased for both carbon sources with the addition of $\mathrm{NaCl}$ and was slightly higher for glucose cultures, compared to glycerol cultures. Under all conditions, high concentrations of hydroxyectoine were produced and naturally excreted into the cultivation medium, with less than $1 \%$ of the total ectoines remaining within the cells (Fig. 2). Final product concentrations in

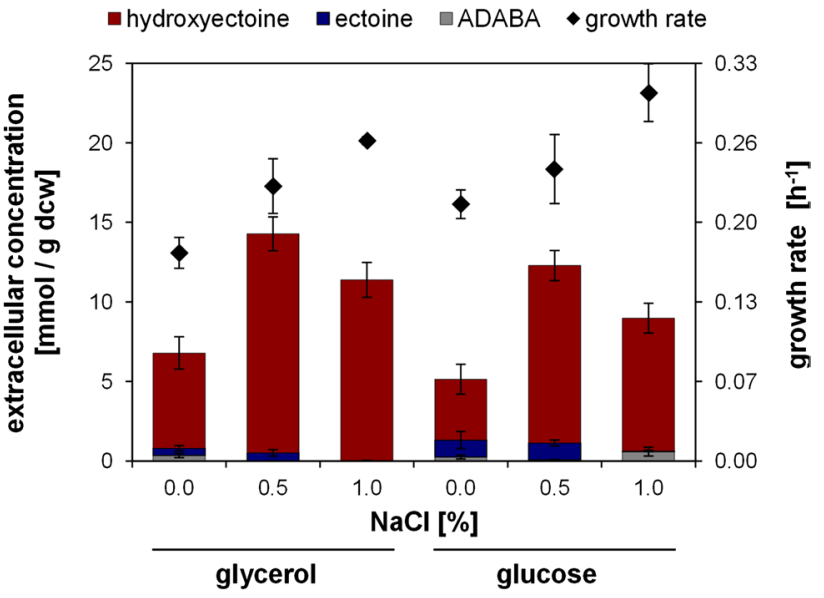

Fig. 2 Hydroxyectoine production in E. coli DH5 $\alpha$ pASK_ectABCDask. The production strain was cultured in MM63 with $0-1 \% \mathrm{NaCl}$ and $50 \mathrm{mM}$ glycerol or $25 \mathrm{mM}$ glucose as carbon source. The specific extracellular content of hydroxyectoine and its precursors (ADABA, ectoine) in $\mathrm{mmol} / \mathrm{g}$ dry cell weight $(\mathrm{dcw})$, as well as the growth rate $\left(\mathrm{h}^{-1}\right)$, were determined. All experiments were performed at least in triplicates. Error bars indicate the standard deviation $(n=3-9)$

the medium ranged from 4.2 to $8.3 \mathrm{mM}$ in glucose cultures and from 5.9 to $10.0 \mathrm{mM}$ in glycerol cultures, depending on the $\mathrm{NaCl}$ content. Thus, glycerol-grown cultures exhibited higher extracellular hydroxyectoine concentrations in all experiments. Besides hydroxyectoine, the precursors ADABA $(<6.5 \%)$ and ectoine $(<20 \%)$ were detected in the medium but no other relevant $\mathrm{N}$-reactive compounds (other than ADABA) nor side products like trehalose were detectable by the HPLC methods employed. The highest specific extracellular hydroxyectoine content of $13.8 \mathrm{mmol} / \mathrm{g}$ $\mathrm{dcw}(2.2 \mathrm{~g} / \mathrm{g} \mathrm{dcw})$ with a low precursor share of $3.6 \%$ was achieved utilizing glycerol medium and $0.5 \% \mathrm{NaCl}$ (Fig. 2). These conditions were the best tested for heterologous hydroxyectoine production in this study.

We then asked whether the presence of the ask gene in the gene cluster had a positive effect on overall hydroxyectoine production. For this purpose, we constructed another production strain, E. coli DH5 $\propto$ pASK_ectABCD, excluding the additional aspartokinase from A. cryptum. However, this strain showed no significant difference in hydroxyectoine production compared to the original production strain ( $E$. coli DH5 $\alpha$ pASK_ectABCDask) (Figure S3, Supplementary material). Thus, the presence of the ask gene from $A$. cryptum had only a marginally beneficial effect on hydroxyectoine production under the conditions employed.

\section{Ectoine production}

During strain construction for hydroxyectoine overproduction, one strain was found to have a random point mutation in $e c t D$. The mutation resulted from the substitution 


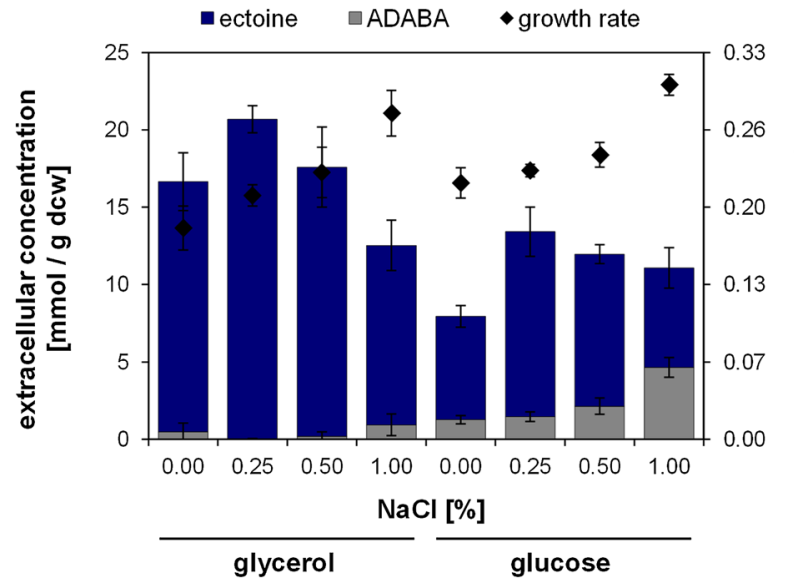

Fig. 3 Ectoine production in E. coli $\mathrm{DH} 5 \alpha \mathrm{pASK}$ ectABCD $\mathrm{m}$. The production strain was cultured in MM63 with $0-1 \% \mathrm{NaCl}$ and $50 \mathrm{mM}$ glycerol or $25 \mathrm{mM}$ glucose as carbon source. The specific extracellular content of ectoine and its precursor ADABA in mmol/g dry cell weight (dcw), as well as the growth rate $\left(\mathrm{h}^{-1}\right)$, were determined. All experiments were performed at least in triplicates. Error bars indicate the standard deviation $(n=3-20)$

of leucine to proline at position 118 in the EctD protein. Experiments showed that this strain, called E. coli DH5 $\alpha$ pASK_ectABCD ${ }_{m}$, was not able to produce hydroxyectoine, but accumulates ectoine in high amounts $(\geq 7 \mathrm{mM})$ (Figure S3, Supplementary material). This strain was therefore used for ectoine overproduction experiments in this study. Later, it was confirmed that $E$. coli DH5 $\alpha$ expressing only the genes ect $A B C$ revealed no significant difference in ectoine production and growth (data not shown).

Ectoine production was performed under the same conditions as described for hydroxyectoine production, except for an additional $\mathrm{NaCl}$ concentration $(0.25 \%)$ in order to further specify the process optimum (Fig. 3). Again, the growth rates were higher for the glucose cultures and increased with increasing $\mathrm{NaCl}$ concentrations. As with hydroxyectoine, high concentrations of ectoine were naturally excreted into the culture medium, and only small amounts $(\leq 1 \%)$ were detected within the cells under optimized conditions. Ectoine concentrations in the medium ranged from 5.5 to $9.2 \mathrm{mM}$ in glucose cultures and from 8.7 to $11.7 \mathrm{mM}$ in glycerol cultures. Furthermore, only small amounts of the precursor ADABA were detected in glycerol-grown cultures $(0-7 \%)$, whereas in glucose-grown cultures the extracellular ADABA concentration was significantly higher (11-42\%). Again, relevant N-reactive compounds other than ADABA, or side products like trehalose, were not detected by the HPLC methods employed. The remarkable maximum specific extracellular ectoine content of $20.6 \mathrm{mmol} / \mathrm{g} \mathrm{dcw}$ $(2.9 \mathrm{~g} / \mathrm{g} \mathrm{dcw})$ was achieved in medium with glycerol and $0.25 \% \mathrm{NaCl}$ (Fig. 3). Under these conditions, no precursor or by-products were detected in the supernatant. Similar to
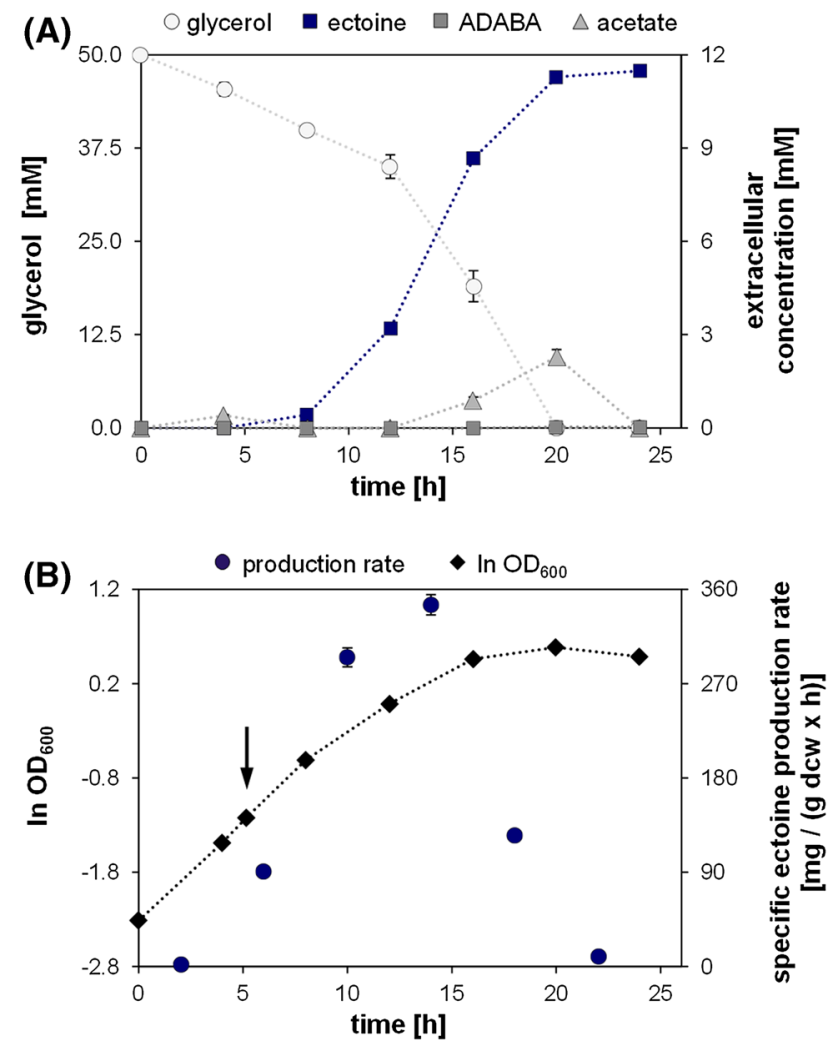

Fig. 4 Ectoine production process over time in E. coli DH5 $\alpha$ pASK ectABCD $D_{m}$. The production strain was cultured in MM63 supplemented with $0.25 \% \mathrm{NaCl}$ and $50 \mathrm{mM}$ glycerol. a Extracellular accumulation of ectoine and side products, as well as glycerol depletion, were determined over time. b The natural logarithm of the growth curve as obtained from the optical density at $600 \mathrm{~nm}\left(\ln \mathrm{OD}_{600}\right)$ is displayed and the time point of induction is indicated by an arrow. The time course of specific ectoine production rate in $\mathrm{mg} /(\mathrm{g} \mathrm{dcw} \times \mathrm{h})$ was approximated by calculating the difference in ectoine concentration at two sampling points divided by the time difference and the mean dry cell weight $(\mathrm{dcw})$. Shown are mean values of two experimental replicates

hydroxyectoine production a low $\mathrm{NaCl}$ concentration and glycerol as carbon source proofed to be optimal for the heterologous production of ectoine in this study.

\section{Specific production rate of ectoines over time}

To characterize the production process of ectoine in our strains in more detail, the specific production rates for $E$. coli DH5 $\alpha$ pASK_ectABCD ${ }_{m}$ were determined. Therefore, samples were taken in 4-h intervals during the ectoine overproduction in shake flask experiments under optimal conditions (MM63 with $0.25 \% \mathrm{NaCl}$ and glycerol). The process was monitored for concentrations of the substrate glycerol and the main product ectoine, as well as for concentrations of the precursor ADABA and the side product acetate (Fig. 4a). After induction, ectoine formation and accumulation in the 
Table 2 Data on heterologous hydroxyectoine and ectoine production efficiency

\begin{tabular}{lll}
\hline & $\begin{array}{l}\text { Hydroxyectoine } \\
\text { producer }(n=9)\end{array}$ & $\begin{array}{l}\text { Ectoine } \\
\text { producer } \\
(n=4)\end{array}$ \\
\hline $\mathrm{NaCl}(\%)$ & 0.50 & 0.25 \\
Growth rate $\left(\mathrm{h}^{-1}\right)$ & $0.23 \pm 0.02$ & $0.21 \pm 0.01$ \\
Carbon source $(\mathrm{g} / \mathrm{L})$ & 4.65 & 4.65 \\
Dry cell weight $(\mathrm{dcw})(\mathrm{g} / \mathrm{L})$ & $0.72 \pm 0.06$ & $0.57 \pm 0.02$ \\
Titer $(\mathrm{g} / \mathrm{L})$ & $1.57 \pm 0.13$ & $1.66 \pm 0.07$ \\
Yield $\mathrm{Y}_{\mathrm{P} / \mathrm{S}}(\mathrm{g} / \mathrm{g})$ & 0.34 & 0.36 \\
Yield $\mathrm{Y}_{(\mathrm{X}+\mathrm{P}) / \mathrm{S}}(\mathrm{g} / \mathrm{g})$ & 0.49 & 0.48 \\
Specific production $(\mathrm{g} / \mathrm{g}$ dcw $)$ & $2.18 \pm 0.17$ & $2.93 \pm 0.37$ \\
Maximum specific production $^{\mathrm{rate}}(\mathrm{mg} /(\mathrm{g}$ dcw $\times \mathrm{h}))$ & $203 \pm 19$ & $345 \pm 1$ \\
${ }^{\text {rate }}$ & & \\
\hline
\end{tabular}

The production values of $E$. coli DH5 $\alpha$ pASK_ectABCDask (hydroxyectoine producer) and $E$. coli $\mathrm{DH} 5 \alpha$ pASK_ectABCD $\mathrm{m}$ (ectoine producer) are shown for optimum conditions using glycerol as carbon source. $\mathrm{Y}_{\mathrm{P} / \mathrm{S}}$ : yield of extracellular hydroxyectoine or ectoine $(\mathrm{P})$ per $\mathrm{g}$ substrate $(\mathrm{S})$ consumed, $\mathrm{Y}_{(\mathrm{X}+\mathrm{P}) / \mathrm{S}}$ : yield of biomass $(\mathrm{X})$ and extracellular hydroxyectoine or ectoine $(\mathrm{P})$ per g substrate $(\mathrm{S})$ consumed

${ }^{\text {a }} n=2$ given as mean with upper/lower boundary

culture medium started and increased rapidly for the next $10 \mathrm{~h}$, until glycerol was depleted. The side product acetate was excreted in moderate amounts $(2.3 \mathrm{mM})$ after $12 \mathrm{~h}$ of the process and was consumed after the main carbon source was no longer available (20-24 h). The precursor ADABA was not detected during the entire process. This confirms previous experiments showing that ADABA is, if at all, only formed in low amounts $(<7 \%)$ when glycerol is used as carbon source. From these data, the time-course of the specific production rate in $\mathrm{mg} /(\mathrm{g} \mathrm{dcw} \times \mathrm{h})$ could be determined, which reached peak values at the late exponential growth phase (Fig. 4b). The maximum specific production rate for the ectoine production process was $345 \mathrm{mg} /(\mathrm{g} \mathrm{dcw} \times \mathrm{h})$. In comparison, the hydroxyectoine production with $E$. coli DH5 $\alpha$ pASK_ectABCDask under optimal conditions resulted in a specific production rate of $203 \mathrm{mg} /(\mathrm{g} \mathrm{dcw} \times \mathrm{h})$ (Table 2).

\section{Carbon balance and maximum yield}

As shown in Table 2 our production strains produced considerably more ectoines than biomass, e.g. E. coli DH5 $\alpha$ pASK_ectABCD ${ }_{m}$ reached almost $3 \mathrm{~g}$ of ectoine per $\mathrm{g} d \mathrm{dcw}$. To elucidate how much carbon source is involved in the heterologous production of ectoines and how much contributes to the formation of biomass, a separated carbon balance was calculated.

Considering the sum of biomass and ectoines as the product, the production strains $E$. coli DH5 $\alpha$ pASK_ectABCD and pASK_ectABCDask reached a yield of $0.48 \mathrm{~g}$ and
0.49 g per g glycerol $\left(\mathrm{Y}_{(\mathrm{X}+\mathrm{P}) / \mathrm{S}}\right)$, respectively (Table 2$)$. These values are significantly higher than those of the control strain $E$. coli DH5 $\alpha$ with the empty vector $(0.38 \mathrm{~g} / \mathrm{g})$, generating only biomass under the same conditions. This means carbon is channelled very efficiently into the formation of ectoines in our production strains.

To separate the carbon input for the biosynthesis of ectoines from the biomass formation, the results of the nonproducing control strain were used as a reference. Here, the control strain required $2.66 \mathrm{~g}$ glycerol (equivalent to $1.04 \mathrm{~g}$ carbon) to form $1 \mathrm{~g}$ of dry biomass. The hydroxyectoine production strain yielded $0.72 \mathrm{~g}$ dry biomass and $1.57 \mathrm{~g}$ extracellular hydroxyectoine from $4.65 \mathrm{~g}$ glycerol (1.82 g carbon). Since we know from our control strain that $0.75 \mathrm{~g}$ carbon are required to generate $0.72 \mathrm{~g}$ dry biomass, the remaining $1.07 \mathrm{~g}$ carbon were channelled into hydroxyectoine production in $E$. coli DH5 $\alpha$ pASK_ectABCDask. From this $0.71 \mathrm{~g}(66 \%)$ carbon could be found directly in the extracellular hydroxyectoine. On a molar basis, this corresponds to a consumption of $30 \mathrm{mmol}$ glycerol for the production of $10 \mathrm{mmol}$ hydroxyectoine. The same ratio applies to the ectoine production strain. Since the carbon of two glycerol molecules is directly channelled into ectoine and hydroxyectoine, respectively, one molecule of glycerol was metabolized otherwise.

The formation of one ectoine molecule $(142.2 \mathrm{~g} / \mathrm{mol})$ from three glycerol molecules $(3 \times 92.1 \mathrm{~g} / \mathrm{mol})$ results in a yield of $0.51 \mathrm{~g} / \mathrm{g}$ for the heterologous production. The fact, that this value is fairly close to the combined yield $\left(\mathrm{Y}_{(\mathrm{X}+\mathrm{P}) / \mathrm{S}}\right)$ of $0.48 \mathrm{~g} / \mathrm{g}$ reflects once more that most of the carbon is channelled into the product during overproduction with our production strain. This yield relates to $62 \%$ of the theoretical maximum of $0.77 \mathrm{~g} / \mathrm{g}$ for a conversion process from two glycerol molecules and no biomass production. Similar results apply to hydroxyectoine (158.2 g/ mol) with a value of $57 \%$ of the theoretical maximum $(0.86 \mathrm{~g} / \mathrm{g})$.

\section{Impact of EctC overexpression on ectoine production}

As shown in Fig. 3, ectoine overproduction may be hampered by the accumulation of the precursor ADABA, in particular on glucose-containing medium. This points towards a bottleneck for the cyclization reaction catalysed by EctC. In order to improve ectoine production and to reduce extracellular accumulation of the precursor ADABA, we constructed the strain E. coli DH5 $\alpha$ pASK ectAB-RectC overexpressing EctC. For this purpose, the plasmid carried the first two genes ect $A$ and $e c t B$ with no alteration to prior constructs, but an additional tet promoter and an optimized RBS upstream of ectC (Figure 


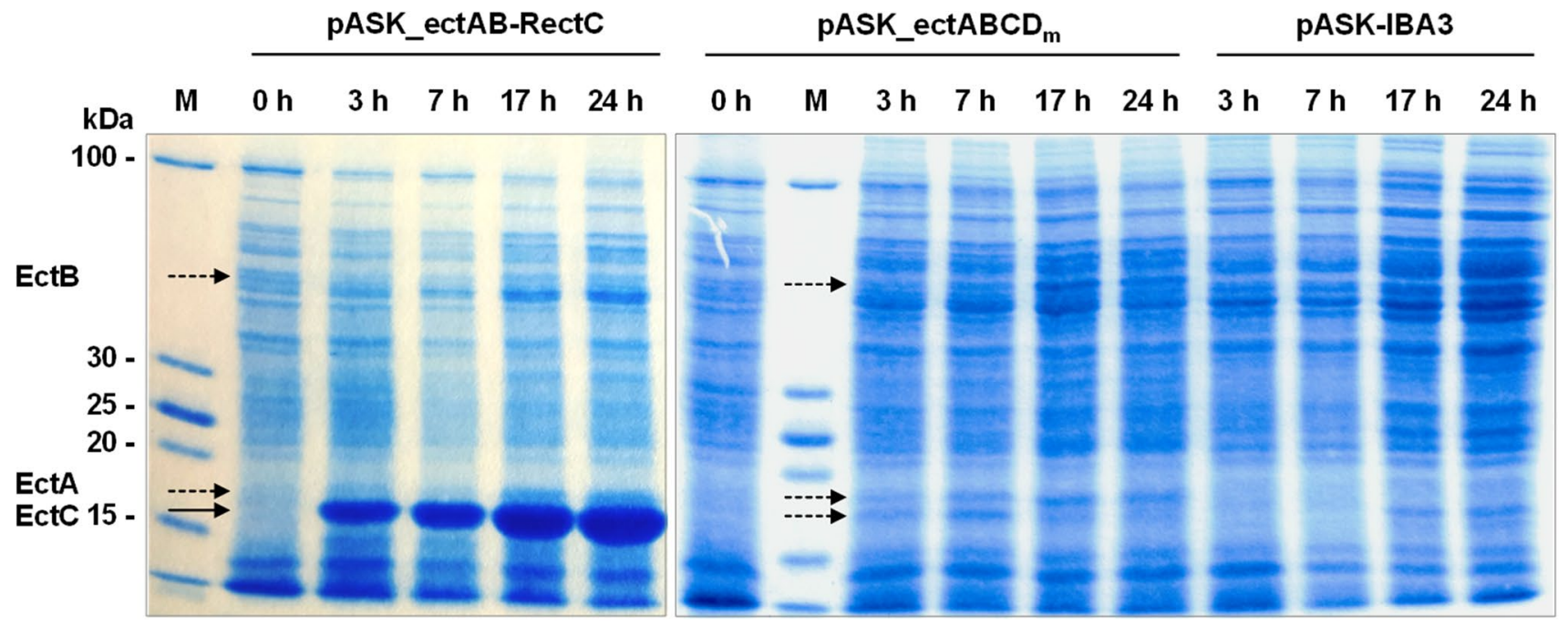

Fig. 5 SDS-PAGE of soluble protein extracts purified from ectoine production strains. Soluble proteins were for peer review isolated from E. coli $\mathrm{DH} 5 \alpha$ carrying the ectoine producing plasmid $\mathrm{pASK}$ ect $\mathrm{ABCD}_{\mathrm{m}}$, the ectC overexpressing plasmid pASK_ectAB-RectC or

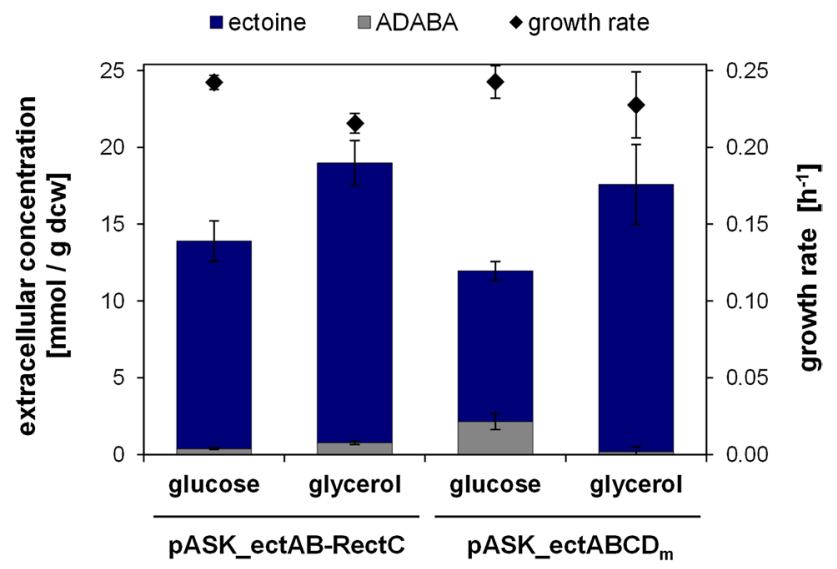

Fig. 6 Impact of EctC overexpression on heterologous ectoine production in E. coli. The EctC overexpressing strain E. coli DH5 $\alpha$ pASK_ectAB-RectC and the ectoine production strain E. coli DH5 $\alpha$ pASK_ectABCDm were cultured in MM63 with $0.5 \% \mathrm{NaCl}$ and $25 \mathrm{mM}$ glucose or $50 \mathrm{mM}$ glycerol as carbon source. The specific extracellular content of ectoine and its precursor ADABA in mmol/g dry cell weight (dcw), as well as the growth rate $\left(\mathrm{h}^{-1}\right)$, were determined. All experiments were performed at least in triplicates. Error bars indicate the standard deviation $(n=3-20)$

S2, Supplementary material). In order to evaluate the protein expression levels of the new plasmid, soluble protein extracts of this strain as well as of the unmodified original ectoine production strain (E. coli DH5 $\alpha$ pASK_ectAB$\mathrm{CD}_{\mathrm{m}}$ ) and a control strain with the empty vector were compared by SDS-PAGE (Fig. 5). The successful overexpression of EctC was clearly demonstrated in protein samples of the new strain, while the EctC protein levels were much lower in the case of $E$. coli $\mathrm{DH} 5 \alpha$ pASK_ectABCD ${ }_{\mathrm{m}}$. the empty vector pASK-IBA3. Samples were taken before induction $(0 \mathrm{~h})$ as well as $3,7,17$, and $24 \mathrm{~h}$ after induction. Expected protein bands are marked by arrows. $M$ protein marker

Subsequently, shake flask experiments were performed to test the ectoine production capability of the EctC overexpression strain (Fig. 6). Cells were grown in minimal medium with either $25 \mathrm{mM}$ glucose or $50 \mathrm{mM}$ glycerol as carbon source. When utilizing glucose, the specific extracellular ectoine content was higher $(13.5 \mathrm{mmol} / \mathrm{g} \mathrm{dcw}$ vs $9.8 \mathrm{mmol} / \mathrm{g} \mathrm{dcw}$ ) and the ADABA proportion significantly lower $(2.8 \%$ vs $18 \%)$ for the EctC overexpression strain, indicating an optimization in the final step of ectoine synthesis by a raised EctC protein level. Similarly, in glycerolgrown cultures the specific extracellular ectoine content was slightly higher $(18.2 \mathrm{mmol} / \mathrm{g} \mathrm{dcw}$ vs $17.4 \mathrm{mmol} / \mathrm{g}$ $\mathrm{dcw}$ ) for the new strain, but the ADABA share $(4.1 \%$ vs $0.01 \%$ ) increased as well (Fig. 6).

\section{Discussion}

\section{The novel approach of utilizing the non-halophilic hydroxyectoine gene cluster from $A$. cryptum for heterologous overproduction of ectoines}

The proteins for hydroxyectoine biosynthesis in A. cryptum exhibit a surprisingly low acidity compared to those of halophilic organisms. In halophiles, an increased amount of acidic amino acids (aspartate, glutamate) in the proteome is an adaptation to high cytoplasmic salt concentrations (Oren et al. 2005; Elevi Bardavid and Oren 2012). This adaptation enables correct folding and activity of enzymes under high salt, but reduces their activity under low salt concentrations (Fukuchi et al. 2003). 


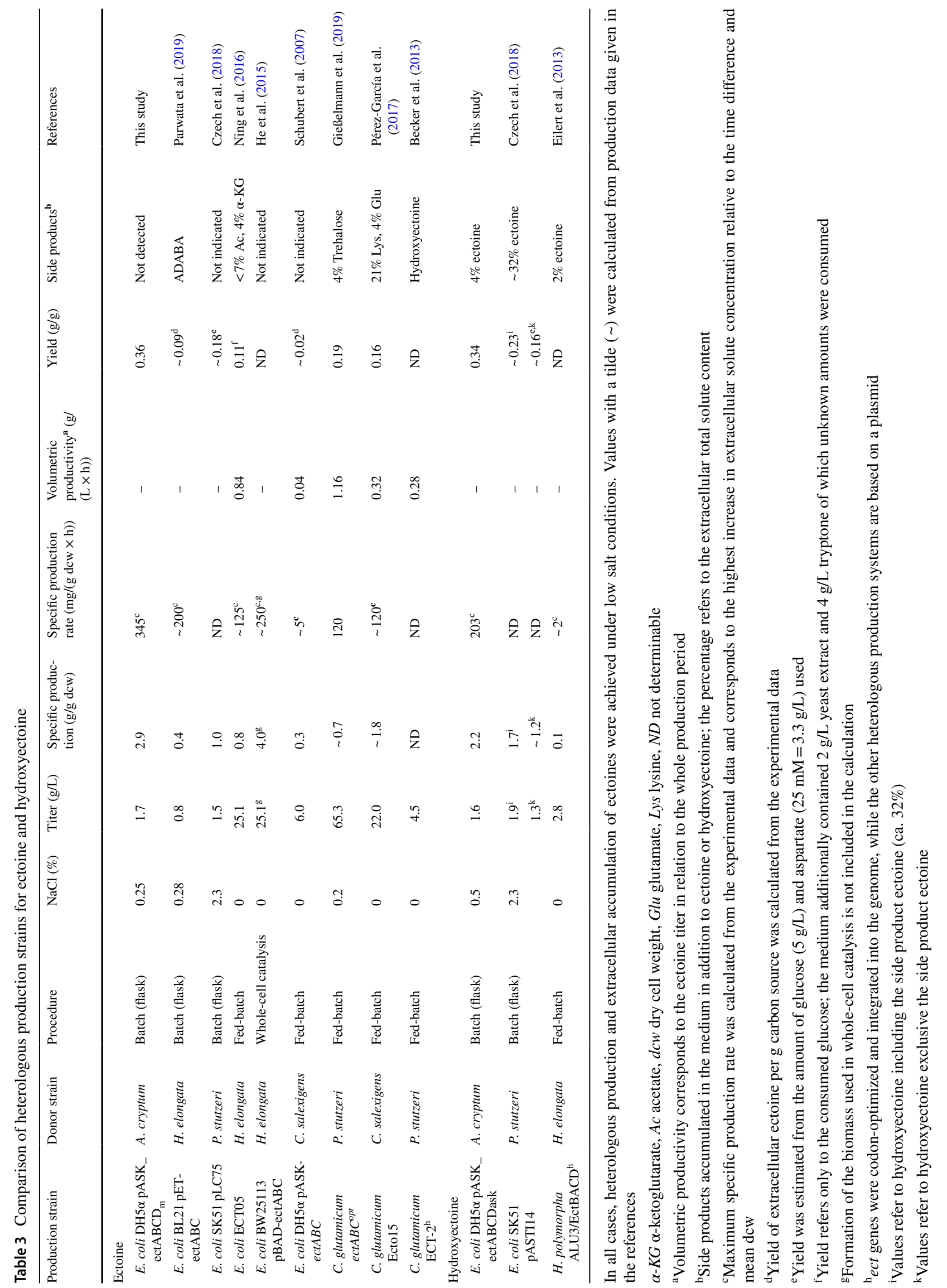


Not surprisingly, therefore only poor activity of the EctC enzyme from the halophilic H. elongata (acidity $=10.2 \%$ ) was recorded under low salt conditions. The activity of the non-halophilic EctC enzyme from A. cryptum (acidity $=3 \%$ ), however, was almost four-times higher at the same low salt conditions (Moritz et al. 2015).

In this study, we were able to confirm the promising attributes of the ectoine/hydroxyectoine biosynthesis proteins from $A$. cryptum for heterologous production processes in E. coli under low salt conditions. We here report the highest specific production (g product/g dcw) of ectoines in a heterologous de-novo synthesis system to date. Expression of the gene cluster under control of a suitable promoter for $E$. coli resulted in high extracellular hydroxyectoine and ectoine concentrations $(\geq 10 \mathrm{mM})$ at low salinities $(0.25-0.5 \% \mathrm{NaCl})$. Interestingly, up to $99 \%$ of the ectoines could be found in the culture supernatant without artificial cell wall perturbation, under the low salt conditions employed. Although efflux of osmolytes at low extracellular ion concentration is apparent, the underlying mechanisms remain unclear. E. coli exhibits several mechanosensitive channels, which allow a rapid efflux of intracellularly accumulated solutes under hypoosmotic conditions to maintain an osmotic equilibrium (Berrier et al. 1996; Levina et al. 1999). These should, however, not be functional in the absence of hypoosmotic stress. Furthermore, there are indications that mechanosensitive channels are not solely responsible, but specific export systems might be involved in the efflux of compatible solutes in E. coli (Czech et al. 2016). In addition, low activity of the osmotically triggered uptake systems ProU and ProP at low salinity might also contribute to the observed accumulation of ectoines in the extracellular space. Although the exact mechanisms of ectoine excretion in E. coli remain unclear, the extracellular accumulation greatly facilitates the down-stream product purification and thereby reduces process costs.

\section{Hydroxyectoine production and influence of aspartokinase from $A$. cryptum}

In this study, we were able to record a specific production of hydroxyectoine of up to $2.2 \mathrm{~g} / \mathrm{g} \mathrm{dcw}$ and a maximum specific production rate of $203 \mathrm{mg} /(\mathrm{g} \mathrm{dcw} \times \mathrm{h})$, using the hydroxyectoine gene cluster from A. cryptum in a heterologous $E$. coli producer strain. To our knowledge, these are the highest values for heterologous hydroxyectoine production systems reported to date (Table 3). Compared to the current industrial production strain $H$. elongata, which requires $\mathrm{NaCl}$ concentrations above $10 \%$ to accumulate hydroxyec-

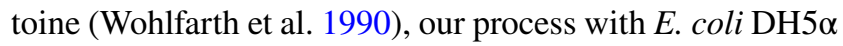
pASK_ectABCDask required only $0.5 \% \mathrm{NaCl}$ and could even be performed without the addition of $\mathrm{NaCl}$. Moreover, in our hydroxyectoine production process, only $3.6 \%$ of the side product ectoine were present in the supernatant of the production strain. This is in contrast to $H$. elongata, which produces hydroxyectoine only alongside high amounts of ectoine, thus resulting in a time-consuming chromatographic separation of both solutes.

Interestingly, a direct comparison of hydroxyectoine production with our production strain using either glucose or glycerol as carbon source revealed a higher product formation under glycerol (4.2-8.3 $\mathrm{mM}$ vs $5.9-10 \mathrm{mM}$ ). This might be explained by the different uptake mechanisms of both carbohydrates in E. coli. While glycerol passes the membrane by facilitated diffusion mediated via GlpF (Sanno et al. 1968), glucose is actively transported by the phosphotransferase system using phosphoenolpyruvate (PEP) as phosphoryl donor (Kundig et al. 1964; Mori and Shiio 1985). As a consequence, high glucose consumption leads to high pyruvate and low PEP levels inside the cell. A low PEP-level is disadvantageous for ectoine production by hindering the resupply of the intermediate oxaloacetate, which is drained from the citrate cycle during the production of ectoines (Ning et al. 2016). The accumulation of pyruvate, though, often leads to unwanted side product formation in microbial production processes (Litsanov et al. 2013). Since E. coli has no effective measures for converting pyruvate directly to oxaloacetate, the unfavourable pyruvate-PEP levels evoked by glucose consumption might therefore be responsible for the lower product formation under glucose compared to glycerol.

As a possible strategy to improve hydroxyectoine production, we included the aspartokinase of A. cryptum into our hydroxyectoine biosynthesis gene cluster for $E$. coli. In 2008, Bestvater et al. had already discovered that the aspartokinase could be a limiting factor for heterologous ectoine production in E. coli. Since then, the incorporation of feedback-resistant aspartokinases has become a common strategy to increase product formation of the aspartate amino acid branch and derivatives, e.g. utilizing LysC from C. glutamicum (Ning et al. 2016). Some organisms already carry an additional ask gene within their ectoine or hydroxyectoine operon, further highlighting its specific role in the production of ectoines. For the ectABCDask gene cluster of Pseudomonas stutzeri A1501 Stöveken et al. (2011) revealed a positive effect of the additional aspartokinase on the intracellular level of hydroxyectoine when expressed heterologously in E. coli. In contrast, Seip et al. (2011) observed no beneficial effect of the aspartokinase using the hydroxyectoine biosynthesis gene cluster from the related P. stutzeri strain DSM $5190^{\mathrm{T}}$. In this study, we achieved only a slight improvement in hydroxyectoine production by including the aspartokinase of the A. cryptum hydroxyectoine biosynthesis gene cluster. Hence, we hesitate to conclude a beneficial effect of the inherent aspartokinase on the heterologous 
production of ectoines in $E$. coli. The already very high production rate in this study demonstrates an already efficient process, which might not be significantly limited in aspartokinase activity. Overall, the here described heterologous production of hydroxyectoine at high efficiency and high purity under low salt concentrations could substantially reduce production costs and therefore allow for new applications with this potent protective substance.

\section{Ectoine production and overexpression of EctC}

Ectoine production with the biosynthetic gene cluster of A. cryptum led to the highest concentrations of $12 \mathrm{mM}$ in the culture supernatant when grown at $0.25 \% \mathrm{NaCl}$. Again, product formation was best when using glycerol as carbon source, leading to a specific production of $2.9 \mathrm{~g} / \mathrm{g} \mathrm{dcw}$ and a maximum specific production rate of $345 \mathrm{mg} /(\mathrm{g} \mathrm{dcw} \times \mathrm{h})$.
To our knowledge, these results are unmatched by any other growth-coupled ectoine production process (Table 3). Our protein analysis further demonstrated that a low general level of heterologous proteins is sufficient to generate these high productions. The low EctD protein concentration may also explain the fact that $E$. coli $\mathrm{DH} 5 \alpha \mathrm{pASK}$ _ectABCD $\mathrm{m}_{\mathrm{m}}$ showed no significant difference in ectoine production and growth compared to the $E$. coli $\mathrm{DH} 5 \alpha$ strain expressing only the ect $A B C$ genes.

One of the most critical points for an efficient ectoine production process is the extracellular accumulation of its precursor ADABA, since its uptake is not possible in $E$. coli (Voß 2002). Consequently, the precursor cannot be reintroduced into ectoine biosynthesis after leaving the cell and an elaborate chromatographic separation would be mandatory for high ectoine purities. In our experiments, the ADABA share on the total product was generally

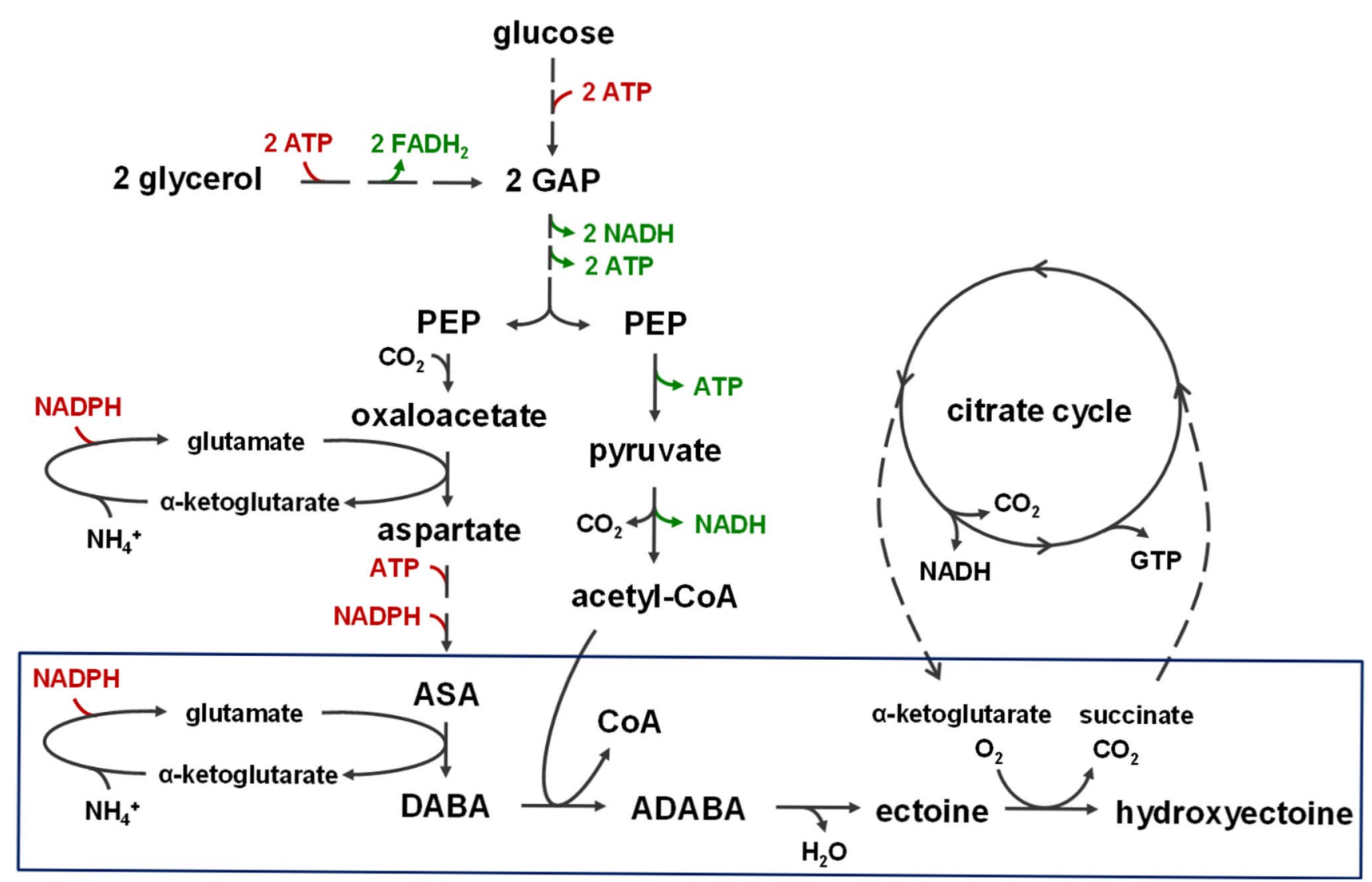

Fig. 7 Energy and co-factor balance of heterologous ectoine and hydroxyectoine biosynthesis in E. coli. The diagram shows the main intermediates of the ectoine and hydroxyectoine formation from glycerol and glucose, respectively, as well as co-factors used and generated on route. The reactions of the heterologous biosynthetic pathway are indicated by a blue box. Ectoine biosynthesis needs one molecule of L aspartate- $\beta$ semialdehyde and acetyl-CoA. In the best-case scenario, oxaloacetate is directly supplied by carboxylation of PEP, while acetyl-CoA is generated from a second PEP. Theoretically, one molecule of glucose or two molecules of glycerol could be sufficient for the generation of one molecule of ectoine. If, however, oxaloacetate is replenished via the glyoxylate cycle, three rather than two molecules of glycerol would be required. In the case of hydroxyectoine production the consumption of $\alpha$-ketoglutarate leads to a shortcut of the citrate cycle, resulting in the loss of $1 \mathrm{NADH}$ and 1 GTP for every turn of the cycle. ASA L-aspartate- $\beta$ semialdehyde, $C o A$ Coenzyme A, $G A P$ glyceraldehyde 3-phosphate, $P E P$ phosphoenolpyruvate 
negligible in culture media containing glycerol as carbon source and even completely absent at $0.25 \% \mathrm{NaCl}$. ADABA formation in glucose cultures, however, was significantly higher and increasing with $\mathrm{NaCl}$ concentration. A possible explanation is that high glucose consumption leads to increased growth rates and to overflow metabolism (Holms 1996), negatively affecting EctC expression or activity. By overexpressing EctC we could solve this problem, confirming a bottleneck in EctC activity for our production strain when grown on glucose. Contrary to glucose, we could observe a slight increase in ADABA formation for the EctC overexpression strain when grown on glycerol. The accumulation in very low amounts $(<5 \%)$ could yet be inevitable due to a reaction equilibrium of $1: 16-20(\leq 6.25 \%$ ADABA) described for EctC of A. cryptum in-vitro (Moritz 2019). Since, the overexpression of EctC could successfully reduce the ADABA accumulation for glucose-grown cultures and could increase the overall specific extracellular ectoine concentration slightly under all conditions tested, EctC expression should be considered for further strain optimizations. Including the optimization of EctC expression, we could generate heterologous strains for ectoine production demonstrating unparalleled specific production grown on glycerol $(2.9 \mathrm{~g} / \mathrm{g} \mathrm{dcw})$ or glucose $(2 \mathrm{~g} / \mathrm{g} \mathrm{dcw})$.

\section{Carbon and energy balance}

In addition to the optimal energy and carbon balance for ectoine biosynthesis with glucose or glycerol in E. coli as depicted in Fig. 7, two molecules of glutamate are needed to supply the amino groups. Assuming that the glutamate dehydrogenase is active in E. coli $\mathrm{DH} 5 \alpha$ under the given growth conditions, one NADPH molecule is required for the regeneration of one glutamate from $\alpha$-ketoglutarate. Following the best-case pathway with glucose as substrate, ATP consumption and production would balance out, while three molecules of NADH are generated and three molecules of NADPH are consumed in biosynthesis. The regeneration of NADPH by NADH, however, requires the input of ATP. When using the more reduced substrate glycerol, two additional molecules of $\mathrm{FADH}_{2}$ are generated, resulting in up to four additional molecules of ATP, which could drive NADPH regeneration.

The carbon balance of our ectoine and hydroxyectoine producer strains revealed that an additional glycerol molecule is consumed during heterologous production in E. coli. Possible reasons are the need for NADPH regeneration, partial use of the glyoxylate cycle for oxaloacetate synthesis, the biosynthesis of the recombinant proteins and maintenance energy needed for a system stressed by overproduction and excretion of products two to three times the amount of its cell mass. Compared to the best-case scenario, the substrate glycerol is very efficiently channelled into ectoine production in the strain we generated.

\section{Comparison of production strains for ectoine and hydroxyectoine}

At present, industrial production of ectoines is primarily performed with the halophilic natural-producer strain $H$. elongata. The production process is facilitated by using ectoine-excreting mutants, so-called leaky mutants (Kunte et al. 2014). Extracellular accumulation of ectoines has the advantages that the cell density does not limit the product titer and that downstream processes can be simplified. Nevertheless, the production of ectoines using $H$. elongata depends on the addition of significant amounts of $\mathrm{NaCl}$, still reducing product revenue. In the case of hydroxyectoine, high amounts of its precursor ectoine are accumulated aside the main product in $H$. elongata, demanding even more elaborate downstream processes. By now, however, already a few heterologous production strains have been developed that achieve accumulation of ectoines in the medium at low salinity or even without the addition of $\mathrm{NaCl}$. For an appropriate comparison with our production strains, we determined the specific production of ectoines, the maximum specific production rate and the yield of ectoines based on the total amount of carbon source consumed, additionally to the product titer (Table 3).

He et al. (2015) performed a whole-cell biocatalysis of ectoine from aspartate and glycerol using $E$. coli with the ectABC gene cluster of $H$. elongata, which yielded a very high specific ectoine production of $4.1 \mathrm{~g} / \mathrm{g} \mathrm{dcw}$. However, since this is the only whole-cell biocatalysis process described, these results are not directly comparable with the specific production of the other de-novo production systems. The formation of the starting biomass used in this whole-cell biocatalysis has not been included in the calculation, leading to the high specific production values. Furthermore, in order to produce $4.1 \mathrm{~g} / \mathrm{g} \mathrm{dcw}$ of ectoine more than $0.35 \mathrm{M}$ of the expensive precursor aspartate $(>46 \mathrm{~g} / \mathrm{L})$ were consumed, in addition to $0.2 \mathrm{M}$ glycerol $(>18 \mathrm{~g} / \mathrm{L})$. This equals a low substrate yield of the process.

Schubert et al. (2007) described the first heterologous ectoine production and excretion by $E$. coli using a culture medium without the addition of $\mathrm{NaCl}$. Despite using a very similar host system (E. coli DH5 $\alpha$ and pASK-IBA7) a significantly lower specific production and maximum specific production rate were reached, compared to our strain E. coli DH5 $\alpha$ pASK_ectABCD ${ }_{\mathrm{m}}$. This might be connected to the ectABC gene cluster from the halophilic bacterium Chromohalobacter salexigens Schubert et al. utilized.

Gießelmann et al. (2019) generated a C. glutamicum strain with the ectoine biosynthesis genes from P. stutzeri 
using a monocistronic approach with balanced transcription of the individual ectoine synthesis genes. The optimal relative expression of the heterologous enzymes was analysed by generating and screening a transcription library. In a fed-batch fermentation with sugar and molasses as carbon sources, the production strain achieved a remarkable ectoine titer of $65.3 \mathrm{~g} / \mathrm{L}$, which is the highest value reported so far. The authors also described a promising specific production rate of $120 \mathrm{mg} /(\mathrm{g} \mathrm{dcw} \times \mathrm{h})$. These data show that a halophilic gene cluster can work well in the Gram-positive organism C. glutamicum.

A high ectoine titer of up to $25.1 \mathrm{~g} / \mathrm{L}$ was obtained by Ning et al. (2016) with an E. coli strain carrying the ectABC operon from $H$. elongata 1A01717 under control of the IPTG-inducible trc promoter. The productivity was increased through several metabolic engineering steps, e.g. overproduction of the PEP carboxylase reinforcing the oxaloacetate pool, and expression of a feedback-resistant aspartokinase from $C$. glutamicum. Overall metabolic engineering resulted in a good maximum ectoine production rate of approx. $125 \mathrm{mg} /$ $(\mathrm{g} \mathrm{dcw} \times \mathrm{h})$ during fed-batch fermentation with complex medium and glucose as the main carbon source.

Compared with the (de-novo) production systems described so far in the literature, our ectoine production strain achieves the highest specific production, production rate and yield, but is limited in product titer. For industrial relevance, the novel and unique potentials of the non-halophilic gene cluster from A. cryptum need to be transferrable into upscaled bioreactor processes.

For hydroxyectoine production only two other heterologous production systems have been described to date, which enable an extracellular hydroxyectoine accumulation at low $\mathrm{NaCl}$ concentrations (Table 3 ).

Eilert et al. (2013) utilized the yeast Hansenula polymor$p h a$ as host for heterologous hydroxyectoine production. The genes ect $A B C D$ from $H$. elongata were codon-optimized and integrated into the genome of $H$. polymorpha. During fedbatch fermentation with methanol, glycerol, and sorbitol as carbon sources, a hydroxyectoine titer of $2.8 \mathrm{~g} / \mathrm{L}$ could be reached with a low ectoine share of $2 \%$. However, the specific hydroxyectoine production and maximum specific production rate were very low when compared to $E$. coli DH5 $\alpha$ pASK_ectABCDask.

Czech et al. (2018) generated an E. coli strain extracellularly accumulating hydroxyectoine as the main product. The highest specific hydroxyectoine production of approx. $1.2 \mathrm{~g} / \mathrm{g} \mathrm{dcw}$ was achieved after several genetic modification steps and by using a culture medium with $2.3 \%$ $\mathrm{NaCl}$ and glucose as well as aspartate as substrate. Under the optimal conditions, a mixture of hydroxyectoine and ectoine with a precursor share of $32 \%$ was accumulated in the culture medium ( $1.9 \mathrm{~g} / \mathrm{L}$ total ectoines). In contrast, our hydroxyectoine-excreting $E$. coli strain reached almost twice the specific hydroxyectoine production with a lower share of the precursor ectoine (4\%).

In this manuscript we are the first to report an ectoine and hydroxyectoine production based on the biosynthesis gene cluster of a non-halophilic bacterium in E. coli. The strains generated achieve unsurpassed specific production and production rates at low $\mathrm{NaCl}$ concentrations and from cheap carbon sources. We believe that the first-ever use of a non-halophilic gene cluster for heterologous overproduction of ectoines may be accountable for this success and should be of pronounced interest for efficient heterologous production of ectoines in E. coli. A more detailed investigation of the performance differences of halophilic and non-halophilic gene cluster for heterologous production would be of interest for upcoming studies. The product titers and cell densities in the here presented shake flask experiments are at a basal level and should be upscaled in the next steps. The data presented, however, already demonstrate an impressive potential of the employed nonhalophilic gene cluster in E. coli. For an industrial application additional improvement is necessary, e.g. the use of a suitable $E$. coli base-strain for industrial production, as well as metabolic engineering and optimization of process conditions. Until this point however, this novel attempt for the heterologous production of ectoines at low $\mathrm{NaCl}$ concentration was rewarded with a benchmark for specific production and production rates. Furthermore, the produced ectoines are mainly accumulated in the medium $(\geq 99 \%$ ), and the proportion of unwanted side products is minimal $(\leq 4 \%)$. Because of these benefits, the use of the non-halophilic gene cluster presented in this study could open new opportunities for the industrial production and purification process of ectoines.

Acknowledgements Open Access funding provided by Projekt DEAL. We would like to thank Erwin Galinski (Institute of Microbiology and Biotechnology, Bonn, Germany) for inspiring discussions and critical reading of the manuscript. We are grateful to Marcel Hövels (Institute of Microbiology and Biotechnology, Bonn, Germany) for kindly providing a spell-checking program.

Author contributions KDM designed the experiments. LB generated the plasmids pASK_ectABCDask, pASK_ectABCD and pASK_ectABC. KDM performed all other experiments and analysed the data. LB and KDM wrote and approved the manuscript.

Funding Financial support for this study was provided by the Institute of Microbiology and Biotechnology of the University Bonn.

\section{Compliance with ethical standards}

Conflict of interest The authors declare that they have no conflict of interest.

Ethical approval This article does not contain any studies with human participants or animals performed by any of the authors. 
Open Access This article is licensed under a Creative Commons Attribution 4.0 International License, which permits use, sharing, adaptation, distribution and reproduction in any medium or format, as long as you give appropriate credit to the original author(s) and the source, provide a link to the Creative Commons licence, and indicate if changes were made. The images or other third party material in this article are included in the article's Creative Commons licence, unless indicated otherwise in a credit line to the material. If material is not included in the article's Creative Commons licence and your intended use is not permitted by statutory regulation or exceeds the permitted use, you will need to obtain permission directly from the copyright holder. To view a copy of this licence, visit http://creativecommons.org/licenses/by/4.0/.

\section{References}

Abdel-Aziz H, Wadie W, Abdallah DM, Lentzen G, Khayyal MT (2013) Novel effects of ectoine, a bacteria-derived natural tetrahydropyrimidine, in experimental colitis. Phytomedicine 20:585591. https://doi.org/10.1016/j.phymed.2013.01.009

Abdel-Aziz H, Wadie W, Scherner O, Efferth T, Khayyal MT (2015) Bacteria-derived compatible solutes ectoine and $5 \alpha$-hydroxyectoine act as intestinal barrier stabilizers to ameliorate experimental inflammatory bowel disease. J Nat Prod 78:1309-1315. https://doi.org/10.1021/acs.jnatprod.5b00115

Bazazzadegan N, Shasaltaneh MD, Saliminejad K, Kamali K, Banan M, Nazari R, Riazi GH, Khorshid HRK (2017) Effects of ectoine on behavior and candidate genes expression in ICV-STZ rat model of sporadic Alzheimer's disease. Adv Pharm Bull 7:629-636. https://doi.org/10.15171/apb.2017.075

Becker J, Wittmann C (2020) Microbial production of extremolyteshigh-value active ingredients for nutrition, health care, and wellbeing. Curr Opin Biotech 65:118-128. https://doi.org/10.1016/j. copbio.2020.02.010

Becker J, Schäfer R, Kohlstedt M, Harder BJ, Borchert NS, Stöveken N, Bremer E, Wittmann C (2013) Systems metabolic engineering of Corynebacterium glutamicum for production of the chemical chaperone ectoine. Microb Cell Fact 12:110. https://doi. org/10.1186/1475-2859-12-110

Berrier C, Besnard M, Ajouz B, Coulombe A, Ghazi A (1996) Multiple mechanosensitive ion channels from Escherichia coli, activated at different thresholds of applied pressure. J Membr Biol 151:175187. https://doi.org/10.1007/s002329900068

Bertani G (1951) Studies on lysogenesis. I. The mode of phage liberation by lysogenic Escherichia coli. J Bacteriol 62:293-300

Bestvater T, Louis P, Galinski EA (2008) Heterologous ectoine production in Escherichia coli: by-passing the metabolic bottle-neck. Saline Syst 4:12. https://doi.org/10.1186/1746-1448-4-12

Bligh EG, Dyer WJ (1959) A rapid method of total lipid extraction and purification. Can J Biochem Physiol 37:911-917. https://doi. org/10.1139/059-099

Borges N, Ramos A, Raven ND, Sharp RJ, Santos H (2002) Comparative study of the thermostabilizing properties of mannosylglycerate and other compatible solutes on model enzymes. Extremophiles 6:209-216. https://doi.org/10.1007/s007920100236

Brown AD (1976) Microbial water stress. Bacteriol Rev 40:803-846

Bursy J, Pierik AJ, Pica N, Bremer E (2007) Osmotically induced synthesis of the compatible solute hydroxyectoine is mediated by an evolutionarily conserved ectoine hydroxylase. J Biol Chem 282:31147-31155. https://doi.org/10.1074/jbc.M704023200

Czech L, Stöveken N, Bremer E (2016) EctD-mediated biotransformation of the chemical chaperone ectoine into hydroxyectoine and its mechanosensitive channel-independent excretion. Microb Cell Fact 15:126. https://doi.org/10.1186/s12934-016-0525-4
Czech L, Poehl S, Hub P, Stöveken N, Bremer E (2018) Tinkering with osmotically controlled transcription allows enhanced production and excretion of ectoine and hydroxyectoine from a microbial cell factory. Appl Environ Microbiol 84:e01772-e1817. https://doi. org/10.1128/AEM.01772-17

Czech L, Höppner A, Kobus S, Seubert A, Riclea R, Dickschat JS, Heider J, Smits SHJ, Bremer E (2019) Illuminating the catalytic core of ectoine synthase through structural and biochemical analysis. Sci Rep 9:364. https://doi.org/10.1038/s41598-018-36247-w

Dinnbier U, Limpinsel E, Schmid R, Bakker EP (1988) Transient accumulation of potassium glutamate and its replacement by trehalose during adaptation of growing cells of Escherichia coli K-12 to elevated sodium chloride concentrations. Arch Microbiol 150:348-357. https://doi.org/10.1007/BF00408306

Eilert E, Kranz A, Hollenberg CP, Piontek M, Suckow M (2013) Synthesis and release of the bacterial compatible solute 5-hydroxyectoine in Hansenula polymorpha. J Biotechnol 167:85-93. https:// doi.org/10.1016/j.jbiotec.2013.02.005

Elevi Bardavid R, Oren A (2012) The amino acid composition of proteins from anaerobic halophilic bacteria of the order Halanaerobiales. Extremophiles 16:567-572. https://doi.org/10.1007/s0079 2-012-0455-y

Follmann M, Becker M, Ochrombel I, Ott V, Krämer R, Marin K (2009) Potassium transport in Corynebacterium glutamicum is facilitated by the putative channel protein $\mathrm{CglK}$, which is essential for $\mathrm{pH}$ homeostasis and growth at acidic $\mathrm{pH}$. J Bacteriol 191:2944-2952. https://doi.org/10.1128/JB.00074-09

Fukuchi S, Yoshimune K, Wakayama M, Moriguchi M, Nishikawa K (2003) Unique amino acid composition of proteins in halophilic bacteria. J Mol Biol 327:347-357. https://doi.org/10.1016/S0022 -2836(03)00150-5

Galinski EA, Herzog RM (1990) The role of trehalose as a substitute for nitrogen-containing compatible solutes (Ectothiorhodospira halochloris). Arch Microbiol 153(6):607-613. https:// doi.org/10.1007/BF00245273

Galinski EA, Oren A (1991) Isolation and structure determination of a novel compatible solute from the moderately halophilic purple sulfur bacterium Ectothiorhodospira marismortui. Eur J Biochem 198:593-598. https://doi.org/10.1111/j.1432-1033.1991.tb160 55. $\mathrm{x}$

Gießelmann G, Dietrich D, Jungmann L, Kohlstedt M, Jeon EJ, Yim SS, Sommer F, Zimmer D, Mühlhaus T, Schroda M, Jeong KJ, Becker J, Wittmann C (2019) Metabolic engineering of Corynebacterium glutamicum for high-level ectoine production: design, combinatorial assembly, and implementation of a transcriptionally balanced heterologous ectoine pathway. Biotechnol J 14:1800417. https://doi.org/10.1002/biot.201800417

Göller K, Galinski EA (1999) Protection of a model enzyme (lactate dehydrogenase) against heat, urea and freeze-thaw treatment by compatible solute additives. J Mol Catal B Enzym 7:37-45. https ://doi.org/10.1016/S1381-1177(99)00043-0

Hanahan D (1983) Studies on transformation of Escherichia coli with plasmids. J Mol Biol 166:557-580. https://doi.org/10.1016/S0022 -2836(83)80284-8

Harrison AP (1981) Acidiphilium cryptum gen. nov., sp. nov., heterotrophic bacterium from acidic mineral environments. Int J Syst Bacteriol 31:327-332. https://doi.org/10.1099/00207 713-31-3-327

He YZ, Gong J, Yu HY, Tao Y, Zhang S, Dong ZY (2015) High production of ectoine from aspartate and glycerol by use of wholecell biocatalysis in recombinant Escherichia coli. Microb Cell Fact 14:55. https://doi.org/10.1186/s12934-015-0238-0

Holms H (1996) Flux analysis and control of the central metabolic pathways in Escherichia coli. FEMS Microbiol Rev 19:85-116. https://doi.org/10.1016/S0168-6445(96)00026-5 
Imhoff JF, Trüper HG (1977) Ectothiorhodospira halochloris sp. nov., a new extremely halophilic phototrophic bacterium containing bacteriochlorophyll b. Arch Microbiol 114:115-121. https://doi. org/10.1007/BF00410772

Kempf B, Bremer E (1998) Uptake and synthesis of compatible solutes as microbial stress responses to high-osmolality environments. Arch Microbiol 170:319-330. https://doi.org/10.1007/s002030050 649

Krämer R, Lambert C, Hoischen C, Ebbighausen H (1990) Uptake of glutamate in Corynebacterium glutamicum. 1. Kinetic properties and regulation by internal $\mathrm{pH}$ and potassium. Eur J Biochem 194:929-935. https://doi.org/10.1111/j.1432-1033.1990.tb194 88. $\mathrm{x}$

Kundig W, Ghosh S, Roseman S (1964) Phosphate bound to histidine in a protein as an intermediate in a novel phospho-transferase system. Proc Natl Acad Sci U S A 52:1067-1074. https://doi. org/10.1073/pnas.52.4.1067

Kunte HJ, Galinski EA, Trüper HG (1993) A modified FMOC-method for the detection of amino acid-type osmolytes and tetrahydropyrimidines (ectoines). J Microbiol Methods 17:129-136. https://doi. org/10.1016/0167-7012(93)90006-4

Kunte HJ, Lentzen G, Galinski EA (2014) Industrial production of the cell protectant ectoine: protection mechanisms, processes, and products. Curr Biotechnol 3:10-25. https://doi.org/10.2174/22115 501113026660037

Laemmli UK (1970) Cleavage of structural proteins during the assembly of the head of bacteriophage T4. Nature 227:680-685. https ://doi.org/10.1038/227680a0

Larsen PI, Sydnes LK, Landfald B, Strøm AR (1987) Osmoregulation in Escherichia coli by accumulation of organic osmolytes: betaines, glutamic acid, and trehalose. Arch Microbiol 147:1. https://doi.org/10.1007/BF00492896

Levina N, Tötemeyer S, Stokes NR, Louis P, Jones MA, Booth IR (1999) Protection of Escherichia coli cells against extreme turgor by activation of MscS and MscL mechanosensitive channels: identification of genes required for MscS activity. EMBO J 18:17301737. https://doi.org/10.1093/emboj/18.7.1730

Lippert K, Galinski EA (1992) Enzyme stabilization by ectoine-type compatible solutes: protection against heating, freezing and drying. Appl Microbiol Biotechnol 37:61-65. https://doi.org/10.1007/ BF00174204

Litsanov B, Brocker M, Bott M (2013) Glycerol as a substrate for aerobic succinate production in minimal medium with Corynebacterium glutamicum. Microb Biotechnol 6:189-195. https://doi.org/ 10.1111/j.1751-7915.2012.00347.x

Louis P, Trüper HG, Galinski EA (1994) Survival of Escherichia coli during drying and storage in the presence of compatible solutes. Appl Microbiol Biotechnol 41:684-688. https://doi.org/10.1007/ BF00167285

Manzanera M, Vilchez S, Tunnacliffe A (2004) High survival and stability rates of Escherichia coli dried in hydroxyectoine. FEMS Microbiol Lett 233:347-352. https://doi. org/10.1111/j.1574-6968.2004.tb09502.x

Mori M, Shiio I (1985) Purification and some properties of phosphoenolpyruvate carboxylase from Brevibacterium flavum and its aspartate-overproducing mutant. J Biochem 97:1119-1128. https ://doi.org/10.1093/oxfordjournals.jbchem.a135156

Moritz KD (2019) Escherichia coli und Synechocystis sp. als heterologe Produktionssysteme für Ectoin und Hydroxyectoin. Dissertation, University Bonn, https://hss.ulb.uni-bonn.de/2019/5336/5336.pdf

Moritz KD, Amendt B, Witt EMHJ, Galinski EA (2015) The hydroxyectoine gene cluster of the non-halophilic acidophile Acidiphilium cryptum. Extremophiles 19:87-99. https://doi.org/10.1007/ s00792-014-0687-0

Ning Y, Wu X, Zhang C, Xu Q, Chen N, Xie X (2016) Pathway construction and metabolic engineering for fermentative production of ectoine in Escherichia coli. Metab Eng 36:10-18. https://doi. org/10.1016/j.ymben.2016.02.013

Oren A, Larimer F, Richardson P, Lapidus A, Csonka LN (2005) How to be moderately halophilic with broad salt tolerance: clues from the genome of Chromohalobacter salexigens. Extremophiles 9:275-279. https://doi.org/10.1007/s00792-005-0442-7

Parwata IP, Wahyuningrum D, Suhandono S, Hertadi R (2019) Heterologous ectoine production in Escherichia coli: optimization using response surface methodology. Int J Microbiol 2019:5475361. https://doi.org/10.1155/2019/5475361

Pastor JM, Salvador M, Argandoña M, Bernal V, Reina-Bueno M, Csonka LN, Iborra JL, Vargas C, Nieto JJ, Cánovas M (2010) Ectoines in cell stress protection: uses and biotechnological production. Biotechnol Adv 28:782-801. https://doi.org/10.1016/j. biotechadv.2010.06.005

Pérez-García F, Ziert C, Risse JM, Wendisch VF (2017) Improved fermentative production of the compatible solute ectoine by Corynebacterium glutamicum from glucose and alternative carbon sources. J Biotechnol 258:59-68. https://doi.org/10.1016/j. jbiotec.2017.04.039

Peters P, Galinski EA, Trüper HG (1990) The biosynthesis of ectoine. FEMS Microbiol Lett 71:157-162. https://doi.org/10.1016/03781097(90)90049-V

Sanno Y, Wilson TH, Lin ECC (1968) Control of permeation to glycerol in cells of Escherichia coli. Biochem Biophys Res Commun 32:344-349. https://doi.org/10.1016/0006-291X(68)90392-6

Schubert T, Maskow T, Benndorf D, Harms H, Breuer U (2007) Continuous synthesis and excretion of the compatible solute ectoine by a transgenic, nonhalophilic bacterium. Appl Environ Microbiol 73:3343-3347. https://doi.org/10.1128/AEM.02482-06

Seip B, Galinski EA, Kurz M (2011) Natural and engineered hydroxyectoine production based on the Pseudomonas stutzeri ect $A B C D$ ask gene cluster. Appl Environ Microbiol 77:1368-1374. https:// doi.org/10.1128/AEM.02124-10

Smith PK, Krohn RI, Hermanson GT, Mallia AK, Gartner FH, Provenzano MD, Fujimoto EK, Goeke NM, Olson BJ, Klenk DC (1985) Measurement of protein using bicinchoninic acid. Anal Biochem 150:76-85. https://doi.org/10.1016/0003-2697(85)90442-7

Stöveken N, Pittelkow M, Sinner T, Jensen RA, Heider J, Bremer E (2011) A specialized aspartokinase enhances the biosynthesis of the osmoprotectants ectoine and hydroxyectoine in Pseudomonas stutzeri A1501. J Bacteriol 193:4456-4468. https://doi. org/10.1128/JB.00345-11

Tanne C, Golovina EA, Hoekstra FA, Meffert A, Galinski EA, Oren A (2014) Glass-forming property of hydroxyectoine is the cause of its superior function as a desiccation protectant. Front Microbiol 5(April):1-13. https://doi.org/10.3389/fmicb.2014.00150

Unfried K, Kroker M, Autengruber A, Gotic M, Sydlik U (2014) The compatible solute ectoine reduces the exacerbating effect of environmental model particles on the immune response of the airways. J Allergy 2014:708458. https://doi.org/10.1155/2014/708458

Voß P (2002) Synthese von kompatiblen Soluten mit ectoinanaloger Struktur und Charakterisierung des protektiven Effektes auf biochemische Modellsysteme und Escherichia coli. Dissertation, Westfälische Wilhelms-Universität Münster, https://d-nb.info/967452503/34

Wohlfarth A, Severin J, Galinski EA (1990) The spectrum of compatible solutes in heterotrophic halophilic eubacteria of the family Halomonadaceae. J Gen Microbiol 136:705-712. https://doi. org/10.1007/BF01955150

Publisher's Note Springer Nature remains neutral with regard to jurisdictional claims in published maps and institutional affiliations. 